\title{
O Uso de Sequências Didáticas no Ensino de Internet das Coisas: uma Experiência no Ensino Médio
}

\author{
André Pinto Moreira \\ Universidade Federal do Paraná \\ Jandaia do Sul, PR, Brasil \\ moreira.andre.p@ufpr.br
}

\author{
Gabriel Jaime Alves \\ Universidade Federal do Paraná \\ Jandaia do Sul, PR, Brasil \\ ga.jalves@ufpr.br
}

\author{
Carlos Roberto Beleti Junior \\ Universidade Federal do Paraná \\ Jandaia do Sul, PR, Brasil \\ carlosbeleti@ufpr.br
}

\author{
Daniela Eloise Flôr \\ Instituto Federal do Paraná \\ Paranavaí, PR, Brasil \\ daniela.flor@ifpr.edu.br
}

\author{
Linnyer Beatrys Ruiz Aylon \\ Universidade Estadual de Maringá \\ Maringá, PR, Brasil \\ Ibruiz@uem.br
}

\begin{abstract}
With the increasing advancement of the Internet and Computing, digital information and communication technologies (DICTs) are increasingly present in everyone's life, whether at work, at home, in cars, or even in clothing, using wearable devices. Then comes the concept Internet of Things (IoT) which aims to interconnect objects with each other and with people through data transmission technologies and various sensors and actuators. With an infrastructure capable of managing them, these objects become "smart". In recent years, there have been increasing efforts to introduce IoT in schools, modifying not only its physical structure but also inserting it as a tool that can enhance interdisciplinary teaching in various activities. Thus, this paper aims to present basic IoT concepts for high school students from a public school in the city of Jandaia do Sul. For this, a didactic sequence was developed, using a smart home model, to demonstrate such concepts. With the sensors of the house, it is possible to demonstrate physics concepts, related to the students' daily life. At the end of the didactic sequence, a questionnaire will be applied to verify the learning process of the students.
\end{abstract}

\section{KEYWORDS}

Internet of Things, Internet das Coisas, Casa Inteligente, Ensino, Sequência Didática.

\section{INTRODUÇÃO}

Com a evolução das Tecnologias Digitais de Informação e Comunicação (TDIC) e das Redes de Sensores Sem Fio (RSSF), o paradigma da Internet das Coisas vem se consolidando nos últimos anos e já é uma realidade no cotidiano das pessoas. A ideia básica deste conceito é a presença generalizada em torno de nós de uma variedade de coisas ou objetos, que, através de esquemas de endereçamento únicos, são capazes de interagir uns com os outros para alcançar objetivos comuns (ATZORI et al., 2010, p. 2787).

Como observado em Araújo e Valentim (2019), Internet das Coisas (IoT - do inglês Internet of Things) "é um paradigma tecnológico que realiza a interconexão entre os mundos físico e digital, criando um 'meio informacional' que aumenta a percepção das pessoas sobre o cenário ao seu redor”.

Atualmente a IoT está presente em ambientes escolares, promovendo mudanças e quebras de paradigmas no modelo educacional tradicional. Segundo Savov et al. (2017), "pesquisadores concordam que a sala de aula deve integrar tecnologias inteligentes e uma pedagogia inovadora”.

\section{Solução Proposta}

Inicialmente, foi realizada uma revisão bibliográfica, com o intuito de verificar quais tecnologias IoT estão sendo utilizadas no Ensino Básico.

Após esta etapa, foram desenvolvidas sequências didáticas, abordando conceitos de IoT, como, por exemplo, um ciclo básico de coleta, transmissão e processamento dos dados obtidos. Também foram abordadas, algumas tecnologias de transmissão de dados, e alguns sensores e atuadores presentes na maquete da Casa Inteligente, conforme visualizado na Figura 1.

Dessa forma, esta proposta baseia-se no ensino de conceitos básicos de IoT para alunos do Ensino Médio, de uma escola pública de Jandaia do Sul. Para isso, serão utilizadas sequencias didáticas, desenvolvidas com base em uma maquete de Casa Inteligente.

A parte frontal da maquete, possui sensores como: ultrassônico HC-SR04, que é utilizado para capturar a presença de objetos, até 
4 metros de distância; o sensor de luminosidade LDR, que varia sua resistência de acordo com a quantidade de luz que é incidida sobre ele; sensor de gás MQ-2; sensor de umidade de solo; sensor de presença PIR - HC-SR501 e um kit RFID MFRC522, utilizado na identificação de objetos por Rádio Frequência.

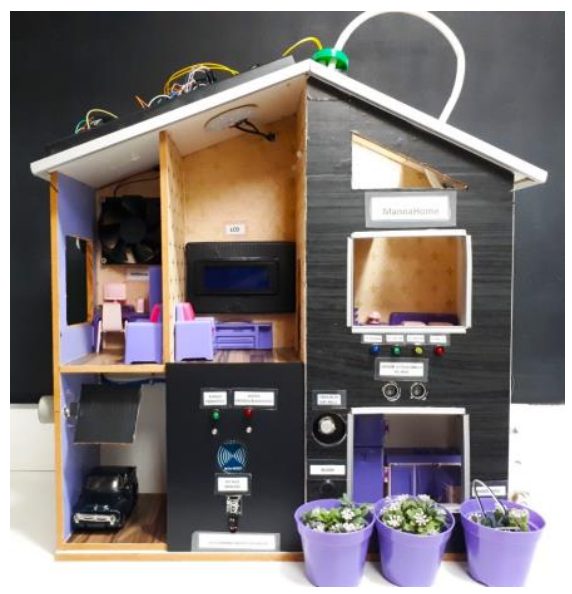

Figura 1: Maquete da Casa Inteligente, visão frontal

Além dos sensores, a maquete possui alguns atuadores como: painel LCD (simulando uma televisão que mostra todas as informações capturadas pelos sensores), luzes de LED, Cooler (simulando um ventilador), motor de passos 28BYJ-48 (simulando um motor elétrico de portão), e buzzer (simulando o alarme).

Os controladores do sistema estão presentes na parte de cima da maquete. Esse sistema é composto por um Arduino Mega, um ESP32 e um ESP8266 NodeMCU. O Arduino é responsável por controlar alguns sensores e as outras duas placas são responsáveis pela comunicação com a Internet.

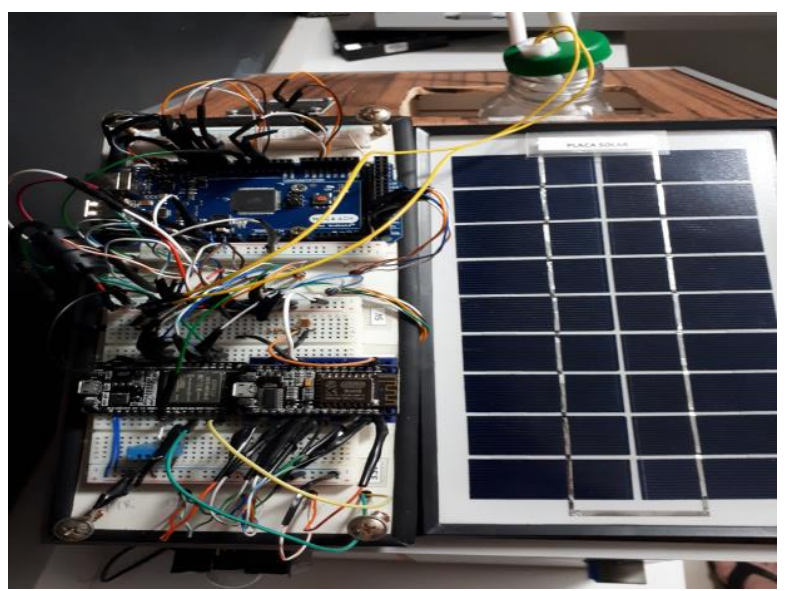

Figura 2: Visão aérea da maquete da Casa Inteligente

A alimentação dos componentes é realizada por meio de um kit off-grid de energia fotovoltaica, composto por uma placa fotovoltaica e um bateria que armazena a energia gerada pela placa, como observado na Figura 2.

Foram desenvolvidas duas sequências didáticas com duração de 120 minutos cada uma. Cada sequência é dividida em duas aulas de 60 minutos. As duas sequencias didáticas se iniciam com a realização de um quiz na primeira aula, com o auxílio de um sistema de resposta à audiência (clickers). Este quiz tem como objetivo verificar os conhecimentos prévio dos alunos, sobre o tema IoT. Após essa dinâmica, são abordados os conceitos básicos de IoT e suas principais tecnologias.

Posteriormente, é apresentada a maquete da Casa Inteligente, o acionamento de seus sensores, demonstrando o seu funcionamento na prática, e a atuação dos sensores são explicados um a um. Para finalizar, a primeira aula das duas sequencias é realizada uma avaliação contendo as perguntas iniciais com intuito de verificar o aprendizado dos alunos durante as atividades.

A segunda aula das duas sequências didáticas é focada nos conceitos de física presente nos sensores. Em uma sequência é abordado o conceito de ondulatória que pode ser trabalhado com o sensor ultrassônico. Na outra sequência, é abordado o efeito fotoelétrico, que pode ser trabalhado com o sensor de luminosidade LDR. Para explicar os dois conceitos físicos são apresentados um vídeo em cada aula com a explicação dos conceitos abordados. Posteriormente, os alunos fazem um experimento em cada sequência didática com relação ao tema proposto em cada uma.

$\mathrm{Na}$ sequência que utiliza ondulatória, os alunos fazem um experimento com o sensor ultrassônico HC-SR04, e na sequência que trata do Efeito Fotoelétrico, os alunos farão um experimento com o sensor de luminosidade LDR, que varia sua resistência conforme a luz que é incidida sobre ele, demonstrando o Efeito Fotoelétrico na prática.

\section{Considerações Finais}

Espera-se que, com este trabalho, seja possível atrair o interesse dos jovens pela IoT, levando em conta que se trata de uma tecnologia emergente que estará presente em grande escala no cotidiano das pessoas em um futuro próximo. Também é esperado que os alunos consigam compreender o funcionamento dos sensores, atuadores, placas e a comunicação com a Internet além dos conceitos presentes na física e que são a base para o funcionamento dos dois sensores utilizados.

\section{REFERÊNCIAS}

[1] Araújo, Carlos Alberto Ávila, e Marta Lígia Pomim Valentim. "A Ciência da Informação no Brasil: mapeamento da pesquisa e cenário institucional." Bibliotecas. Anales de Investigación 15.2 (2019): 323-259. [2] Atzori, Luigi, Antonio Iera, and Giacomo Morabito. "The internet of things: A survey." Computer networks 54.15 (2010): 2787-2805.

[3] Savov, Teodor et al. Contemporany technology support for education. In CBU Internacional Conference Preceedings. 2017. p. 802-806.dsd [4] BORGIA, Eleonora. The Internet of Things vision: Key features, applications and open issues. Computer Communications, v. 54, p. 131, 2014. 